\title{
An enumeration of flags in finite vector spaces
}

\author{
C. Ryan Vinroot* \\ Department of Mathematics \\ College of William and Mary \\ P. O. Box 8795 \\ Williamsburg, VA 23187 \\ vinroot@math.wm.edu
}

Submitted: Feb 2, 2012; Accepted: Jun 27, 2012; Published: Jul 12, 2012

Mathematics Subject Classifications: 05A19, 05A15, 05A30

\begin{abstract}
By counting flags in finite vector spaces, we obtain a $q$-multinomial analog of a recursion for $q$-binomial coefficients proved by Nijenhuis, Solow, and Wilf. We use the identity to give a combinatorial proof of a known recurrence for the generalized Galois numbers.
\end{abstract}

\section{Introduction}

For a parameter $q \neq 1$, and a positive integer $n$, let $(q)_{n}=(1-q)\left(1-q^{2}\right) \cdots\left(1-q^{n}\right)$, and $(q)_{0}=1$. For non-negative integers $n$ and $k$, with $n \geqslant k$, the $q$-binomial coefficient or Gaussian polynomial, denoted $\left(\begin{array}{l}n \\ k\end{array}\right)_{q}$, is defined as $\left(\begin{array}{l}n \\ k\end{array}\right)_{q}=\frac{(q)_{n}}{(q)_{k}(q)_{n-k}}$.

The Rogers-Szegö polynomial in a single variable, denoted $H_{n}(t)$, is defined as

$$
H_{n}(t)=\sum_{k=0}^{n}\left(\begin{array}{l}
n \\
k
\end{array}\right)_{q} t^{k} .
$$

The Rogers-Szegö polynomials first appeared in papers of Rogers [16, 17] which led up to the famous Rogers-Ramanujan identities, and later were independently studied by Szegö [19]. They are important in combinatorial number theory ([1, Ex. 3.3-3.9] and [5, Sec. 20]), symmetric function theory [20], and are key examples of orthogonal polynomials [2]. They also have applications in mathematical physics [11, 13].

The Rogers-Szegö polynomials satisfy the recursion (see [1, p. 49])

$$
H_{n+1}(t)=(1+t) H_{n}(t)+t\left(q^{n}-1\right) H_{n-1}(t) .
$$

\footnotetext{
${ }^{*}$ Supported by NSF grant DMS-0854849.
} 
Letting $t=1$, we have $H_{n}(1)=\sum_{k=0}^{n}\left(\begin{array}{l}n \\ k\end{array}\right)_{q}$, which, when $q$ is the power of a prime, is the total number of subspaces of an $n$-dimensional vector space over a field with $q$ elements. The numbers $G_{n}=H_{n}(1)$ are the Galois numbers, and from (1.1), satisfy the recursion

$$
G_{n+1}=2 G_{n}+\left(q^{n}-1\right) G_{n-1} .
$$

The Galois numbers were studied from the point of view of finite vector spaces by Goldman and Rota [6], and have been studied extensively elsewhere, for example, in [15, 9]. In particular, Nijenhuis, Solow, and Wilf [15] give a bijective proof of the recursion (1.2) using finite vector spaces, by proving, for integers $n \geqslant k \geqslant 1$,

$$
\left(\begin{array}{c}
n+1 \\
k
\end{array}\right)_{q}=\left(\begin{array}{l}
n \\
k
\end{array}\right)_{q}+\left(\begin{array}{c}
n \\
k-1
\end{array}\right)_{q}+\left(q^{n}-1\right)\left(\begin{array}{l}
n-1 \\
k-1
\end{array}\right)_{q}
$$

For non-negative integers $k_{1}, k_{2}, \ldots, k_{m}$ such that $k_{1}+\cdots+k_{m}=n$, we define the $q$-multinomial coefficient of length $m$ as

$$
\left(\begin{array}{c}
n \\
k_{1}, k_{2}, \ldots, k_{m}
\end{array}\right)_{q}=\frac{(q)_{n}}{(q)_{k_{1}}(q)_{k_{2}} \cdots(q)_{k_{m}}},
$$

so that $\left(\begin{array}{l}n \\ k\end{array}\right)_{q}=\left(\begin{array}{c}n \\ k, n-k\end{array}\right)_{q}$. If $\underline{k}$ denotes the $m$-tuple $\left(k_{1}, \ldots, k_{m}\right)$, write the corresponding $q$-multinomial coefficient as $\left(\begin{array}{c}n \\ k_{1}, \ldots, k_{m}\end{array}\right)_{q}=\left(\begin{array}{l}n \\ \underline{k}\end{array}\right)_{q}$. For a subset $J \subseteq\{1, \ldots, m\}$, let $\underline{e}_{J}$ denote the $m$-tuple $\left(e_{1}, \ldots, e_{m}\right)$, where

$$
e_{i}= \begin{cases}1 & \text { if } i \in J \\ 0 & \text { if } i \notin J\end{cases}
$$

For example, if $m=3, J=\{1,3\}$, and $\underline{k}=\left(k_{1}, k_{2}, k_{3}\right)$, then $\left(\begin{array}{c}n \\ \underline{k}-\underline{e}_{J}\end{array}\right)_{q}=\left(\begin{array}{c}n \\ k_{1}-1, k_{2}, k_{3}-1\end{array}\right)_{q}$. The main result of this paper, which is obtained in Section 2, Theorem 2.1, is a combinatorial proof through enumerating flags in finite vectors spaces of the following generalization of the identity (1.3). For $m \geqslant 2$, and any $k_{1}, \ldots, k_{m}>0$ such that $k_{1}+\cdots+k_{m}=n+1$, we have

$$
\left(\begin{array}{c}
n+1 \\
k_{1}, \ldots, k_{m}
\end{array}\right)_{q}=\sum_{J \subseteq\{1, \ldots, m\},|J|>0}(-1)^{|J|-1} \frac{(q)_{n}}{(q)_{n-|J|+1}}\left(\begin{array}{c}
n+1-|J| \\
\underline{k}-\underline{e}_{J}
\end{array}\right)_{q} .
$$

In Section 3, we prove a recursion which generalizes (1.2). In particular, the generalized Galois number $G_{n}^{(m)}$ is defined as

$$
G_{n}^{(m)}=\sum_{k_{1}+\cdots+k_{m}=n}\left(\begin{array}{c}
n \\
k_{1}, k_{2}, \ldots, k_{m}
\end{array}\right)_{q}
$$

which, in the case that $q$ is the power of a prime, enumerates the total number of flags of length $m-1$ of an $n$-dimensional $\mathbb{F}_{q}$-vector space. Quite recently, the asymptotic statistics of these generalized Galois numbers have been studied by Bliem and Kousidis [3] and Kousidis [12]. 
Directly following from Theorem 2.1, we prove in Theorem 3.1 that, for $n \geqslant m-1$,

$$
G_{n+1}^{(m)}=\sum_{i=0}^{m-1}\left(\begin{array}{c}
m \\
i+1
\end{array}\right)(-1)^{i} \frac{(q)_{n}}{(q)_{n-i}} G_{n-i}^{(m)}
$$

which also follows from a known recurrence for the multivariate Rogers-Szegö polynomials.

\section{Flags in finite vector spaces}

In this section, let $q$ be the power of a prime, and let $\mathbb{F}_{q}$ denote a finite field with $q$ elements. If $V$ is an $n$-dimensional vector space over $\mathbb{F}_{q}$, then the $q$-binomial coefficient $\left(\begin{array}{l}n \\ k\end{array}\right)_{q}$ is the number of $k$-dimensional subspaces of $V$ (see [10, Thm. 7.1] or [18, Prop. 1.3.18]). So, the Galois number

$$
G_{n}=H_{n}(1)=\sum_{k=0}^{n}\left(\begin{array}{l}
n \\
k
\end{array}\right)_{q}
$$

is the total number of subspaces of an $n$-dimensional vector space over $\mathbb{F}_{q}$.

Now consider the $q$-multinomial coefficient in terms of vector spaces over $\mathbb{F}_{q}$. It follows from the definition of a $q$-multinomial coefficient and the fact that $\left(\begin{array}{l}n \\ k\end{array}\right)_{q}=\left(\begin{array}{c}n \\ n-k\end{array}\right)_{q}$ that we have

$$
\begin{aligned}
\left(\begin{array}{c}
n \\
k_{1}, k_{2}, \ldots, k_{m}
\end{array}\right)_{q} & =\left(\begin{array}{c}
n \\
k_{1}
\end{array}\right)_{q}\left(\begin{array}{c}
n-k_{1} \\
k_{2}
\end{array}\right)_{q} \ldots\left(\begin{array}{c}
n-k_{1}-\cdots-k_{m-2} \\
k_{m-1}
\end{array}\right)_{q} \\
& =\left(\begin{array}{c}
n \\
n-k_{1}
\end{array}\right)_{q}\left(\begin{array}{c}
n-k_{1} \\
n-k_{1}-k_{2}
\end{array}\right)_{q} \cdots\left(\begin{array}{c}
n-k_{1}-\cdots-k_{m-2} \\
n-k_{1}-\cdots-k_{m-2}-k_{m-1}
\end{array}\right)_{q} .
\end{aligned}
$$

So, if $V$ is an $n$-dimensional vector space over $\mathbb{F}_{q}$, the $q$-multinomial coefficient $\left(\begin{array}{c}n \\ k_{1}, \ldots, k_{m}\end{array}\right)_{q}$ is equal to the number of ways to choose an $\left(n-k_{1}\right)$-dimensional subspace $W_{1}$ of $V$, an $\left(n-k_{1}-k_{2}\right)$-dimensional subspace $W_{2}$ of $W_{1}$, and so on, until finally we choose an $\left(n-k_{1}-\cdots-k_{m-1}\right)$-dimensional subspace $W_{m-1}$ of some $\left(n-k_{1}-\cdots-k_{m-2}\right)$-dimensional subspace $W_{m-2}$ (see also [14, Sec. 1.5]). That is,

$$
W_{m-1} \subseteq W_{m-2} \subseteq \cdots \subseteq W_{2} \subseteq W_{1}
$$

is a flag of subspaces of $V$ of length $m-1$, where $\operatorname{dim} W_{i}=n-\sum_{j=1}^{i} k_{j}$.

We now turn to a bijective proof of the identity (1.3), that for integers $n \geqslant k \geqslant 1$,

$$
\left(\begin{array}{c}
n+1 \\
k
\end{array}\right)_{q}=\left(\begin{array}{l}
n \\
k
\end{array}\right)_{q}+\left(\begin{array}{c}
n \\
k-1
\end{array}\right)_{q}+\left(q^{n}-1\right)\left(\begin{array}{l}
n-1 \\
k-1
\end{array}\right)_{q}
$$

While the bijective interpretation of this identity which we give now is different from the proof given by Nijenhuis, Solow, and Wilf in [15], it is the interpretation which is most 
helpful for the proof of our main result. Fix $V$ to be an $(n+1)$-dimensional $\mathbb{F}_{q}$-vector space. There are $\left(\begin{array}{c}n+1 \\ k\end{array}\right)_{q}$ ways to choose a $k$-dimensional subspace $W$ of $V$. Fix a basis $\left\{v_{1}, v_{2}, \ldots, v_{n+1}\right\}$ of $V$. Any $k$-dimensional subspace $W$ can be written as $\operatorname{span}\left(W^{\prime}, v\right)$ where $W^{\prime}$ is a $(k-1)$-dimensional subspace of $V^{\prime}=\operatorname{span}\left(v_{1}, \ldots, v_{n}\right)$, for some $v$. We may choose $W$ in three distinct ways. If $v \in V^{\prime}$, then $W$ is a subspace of $V^{\prime}$, for which there are $\left(\begin{array}{l}n \\ k\end{array}\right)_{q}$ choices. Call this a type 1 subspace of $V$. If $v_{n+1} \in W$, then we may take $v=v_{n+1}$, and $W$ is determined by $W^{\prime}$, for which there are $\left(\begin{array}{c}n \\ k-1\end{array}\right)_{q}$ choices. We call this a type 2 subspace of $V$. Finally, if both $W \not \subset V^{\prime}$ and $v_{n+1} \notin W$, then we call $W$ a type 3 subspace of $V$, and it follows from (1.3) (and can be shown directly, as well) that there are $\left(q^{n}-1\right)\left(\begin{array}{l}n-1 \\ k-1\end{array}\right)_{q}$ choices for $W$.

We may now prove our main result.

Theorem 2.1. For $m \geqslant 2$, and any $k_{1}, \ldots, k_{m}>0$ such that $k_{1}+\cdots+k_{m}=n+1$, we have

$$
\left(\begin{array}{c}
n+1 \\
k_{1}, \ldots, k_{m}
\end{array}\right)_{q}=\sum_{J \subseteq\{1, \ldots, m\},|J|>0}(-1)^{|J|-1} \frac{(q)_{n}}{(q)_{n-|J|+1}}\left(\begin{array}{c}
n+1-|J| \\
\underline{k}-\underline{e}_{J}
\end{array}\right)_{q}
$$

Proof. Fix $V$ to be an $(n+1)$-dimensional vector space over $\mathbb{F}_{q}$. Fix a basis of each subspace $U$ of $V$, so that we may speak of subspaces of type 1,2 , or 3 of each subspace $U$ with respect to this fixed basis. Consider a flag $F$ of subspaces of $V=W_{0}, W_{m-1} \subset$ $\cdots \subset W_{2} \subset W_{1}$, such that if we define $k_{i}$ for $1 \leqslant i \leqslant m$ by $\sum_{j=1}^{i} k_{j}=n+1-\operatorname{dim} W_{i}$, then each $k_{i}>0$. The total number of such flags is $\left(\begin{array}{c}n+1 \\ k_{1}, \ldots, k_{m}\end{array}\right)_{q}$. Consider now a labeling of such flags in the following way. Given a flag $F$ as above, define

$$
r=\min \left\{1 \leqslant j \leqslant m \mid W_{j} \text { is a type } 1 \text { subspace of } W_{j-1}\right\},
$$

and

$$
J=\{r\} \cup\left\{1 \leqslant j \leqslant r-1 \mid W_{j} \text { is a type } 3 \text { subspace of } W_{j-1}\right\} .
$$

Define the flag $F$ to be a type $J$ flag of $V$. That is, for any nonempty $J \subseteq\{1, \ldots, m\}$, we may speak of flags of type $J$ of $V$. We shall prove that

$$
(-1)^{|J|-1} \frac{(q)_{n}}{(q)_{n-|J|+1}}\left(\begin{array}{c}
n+1-|J| \\
\underline{k}-\underline{e}_{J}
\end{array}\right)_{q}
$$

is the number of type $J$ flags of length $m-1$ of the $\mathbb{F}_{q}$-space $V$. Once this claim is proven, we will have accounted for all $2^{m}-1$ terms on the right-side of the desired result of Theorem 2.1, and all possible ways to choose our flag.

We prove the claim by induction on $m$, where the base case of $m=2$ follows from (1.3) and its interpretation in terms of subspaces of types 1,2 , and 3 , as given above. We must consider each possible nonempty $J \subseteq\{1, \ldots, m\}$, and show that in each case, the quantity (2.1) counts the number of type $J$ flags. So, consider a flag of subspaces $W_{m-1} \subset \cdots \subset W_{2} \subset W_{1}$ of $V$, where $\operatorname{dim} W_{i}=n+1-\sum_{j=1}^{i} k_{j}$.

First, if $J=\{1\}$, then the number of ways to choose $W_{1}$ to be a type 1 subspace of $V$ of dimension $n+1-k_{1}$ is $\left(\begin{array}{c}n \\ n+1-k_{1}\end{array}\right)$, while the number of ways to choose the remaining length 
$m-2$ flag $W_{m-1} \subset \cdots \subset W_{2}$ of $W_{1}$ is exactly $\left(\begin{array}{c}n+1-k_{1} \\ k_{2}, \ldots, k_{m}\end{array}\right)$. Thus, the total number of ways to choose our flag of type $J$ with $J=\{1\}$ is $\left(\begin{array}{c}n \\ n+1-k_{1}\end{array}\right)_{q}\left(\begin{array}{c}n+1-k_{1} \\ k_{2}, \ldots, k_{m}\end{array}\right)_{q}=\left(\begin{array}{c}n \\ k_{1}-1, k_{2}, \ldots, k_{m}\end{array}\right)$, which is exactly the expression (2.1) for $J=\{1\}$, as claimed. So, we now suppose $J \neq\{1\}$, so if $r$ is the maximum element of $J$, we have $r>1$. We consider the cases of whether $1 \in J$ or $1 \notin J$ separately.

Suppose that $1 \notin J$. Then, we must choose our flag so that $W_{1}$ is a type 2 subspace of $V$, of which there are $\left(\begin{array}{c}n \\ n-k_{1}\end{array}\right)_{q}$ such subspaces. Now, if we define $I=J-1=\{j-1 \mid j \in J\}$, so that $I \subset\{1, \ldots, m-1\}$ and $|I|=|J|$, we must choose the rest of our type $J$ flag of $V$ by choosing a type $I$ flag of $W_{1}$ of length $m-2$. If we let $\underline{k}^{\prime}=\left(k_{2}, \ldots, k_{m}\right)$, then by our induction hypothesis, the number of type $I$ flags of length $m-2$ of the $\left(n+1-k_{1}\right)$ dimensional space $W_{1}$ is

$$
(-1)^{|I|-1} \frac{(q)_{n-k_{1}}}{(q)_{n-k_{1}-|I|+1}}\left(\begin{array}{c}
n+1-k_{1}-|I| \\
\underline{k}^{\prime}-\underline{e}_{I}
\end{array}\right)_{q} .
$$

So, the total number of ways to choose the type $J$ flag of length $m-1$ in $V$ is

$$
\left(\begin{array}{c}
n \\
n-k_{1}
\end{array}\right)_{q}(-1)^{|I|-1} \frac{(q)_{n-k_{1}}}{(q)_{n-k_{1}-|I|+1}}\left(\begin{array}{c}
n+1-k_{1}-|I| \\
\underline{k}^{\prime}-\underline{e}_{I}
\end{array}\right)_{q} .
$$

A direct computation yields

$$
\left(\begin{array}{c}
n \\
n-k_{1}
\end{array}\right)_{q} \frac{(q)_{n-k_{1}}}{(q)_{n-k_{1}-|I|+1}}=\frac{(q)_{n}}{(q)_{n-|I|+1}}\left(\begin{array}{c}
n+1-|I| \\
n-k_{1}-|I|+1
\end{array}\right)_{q},
$$

and further note that

$$
\left(\begin{array}{c}
n+1-|I| \\
n-k_{1}-|I|+1
\end{array}\right)_{q}\left(\begin{array}{c}
n+1-k_{1}-|I| \\
\underline{k}^{\prime}-\underline{e}_{I}
\end{array}\right)_{q}=\left(\begin{array}{c}
n+1-|J| \\
\underline{k}-\underline{e}_{J}
\end{array}\right)_{q},
$$

where $\underline{k}=\left(k_{1}, \ldots, k_{m}\right)$. Together, these give

$$
\begin{aligned}
\left(\begin{array}{c}
n \\
n-k_{1}
\end{array}\right)_{q}(-1)^{|I|-1} \frac{(q)_{n-k_{1}}}{(q)_{n-k_{1}-|I|+1}} & \left(\begin{array}{c}
n+1-k_{1}-|I| \\
\underline{k}^{\prime}-\underline{e}_{I}
\end{array}\right)_{q} \\
& =(-1)^{|J|-1} \frac{(q)_{n}}{(q)_{n-|J|+1}}\left(\begin{array}{c}
n+1-|J| \\
\underline{k}-\underline{e}_{J}
\end{array}\right)_{q},
\end{aligned}
$$

giving the claim that when $1 \notin J$, the number of type $J$ subspaces of length $m-1$ of $V$ is given by $(2.1)$.

Finally, suppose that $1 \in J$, and $J \neq\{1\}$. So, we must choose our flag so that $W_{1}$ is a type 3 subspace of $V$, and there are $\left(q^{n}-1\right)\left(\begin{array}{c}n-1 \\ n-k_{1}\end{array}\right)_{q}$ such subspaces. If we let $I=(J-1) \backslash\{0\}$ (so that now $|J|=|I|+1)$, then we must choose the rest of our flag as a type $I$ flag of length $m-2$ of $W_{1}$. Letting again $\underline{k}^{\prime}=\left(k_{2}, \ldots, k_{m}\right)$, then by our induction hypothesis, the total number of flags of type $J$ of length $m-1$ of $V$ is given by

$$
\left(q^{n}-1\right)\left(\begin{array}{c}
n-1 \\
n-k_{1}
\end{array}\right)_{q}(-1)^{|I|-1} \frac{(q)_{n-k_{1}}}{(q)_{n-k_{1}-|I|+1}}\left(\begin{array}{c}
n+1-k_{1}-|I| \\
\underline{k}^{\prime}-\underline{e}_{I}
\end{array}\right)_{q} .
$$


A computation gives

$$
\left(q^{n}-1\right)\left(\begin{array}{c}
n-1 \\
n-k_{1}
\end{array}\right)_{q} \frac{(q)_{n-k_{1}}}{(q)_{n-k_{1}-|I|+1}}=(-1) \frac{(q)_{n}}{(q)_{n-|I|}}\left(\begin{array}{c}
n-|I| \\
n-k_{1}-|I|+1
\end{array}\right)_{q},
$$

and also note

$$
\left(\begin{array}{c}
n-|I| \\
n-k_{1}-|I|+1
\end{array}\right)_{q}\left(\begin{array}{c}
n+1-k_{1}-|I| \\
\underline{k}^{\prime}-\underline{e}_{I}
\end{array}\right)_{q}=\left(\begin{array}{c}
n+1-|J| \\
\underline{k}-\underline{e}_{J}
\end{array}\right)_{q},
$$

where $\underline{k}=\left(k_{1}, \ldots, k_{m}\right)$, since $|I|=|J|-1$. We finally obtain that

$$
\begin{aligned}
\left(q^{n}-1\right)\left(\begin{array}{c}
n-1 \\
n-k_{1}
\end{array}\right)_{q}(-1)^{|I|-1} \frac{(q)_{n-k_{1}}}{(q)_{n-k_{1}-|I|+1}}\left(\begin{array}{c}
n+1-k_{1}-|I| \\
\underline{k}^{\prime}-\underline{e}_{I}
\end{array}\right)_{q} & \\
& =(-1)^{|J|-1} \frac{(q)_{n}}{(q)_{n-|J|+1}}\left(\begin{array}{c}
n+1-|J| \\
\underline{k}-\underline{e}_{J}
\end{array}\right)_{q},
\end{aligned}
$$

is the the number of type $J$ subspaces of length $m-1$ of $V$, as claimed.

\section{Generalized Galois numbers}

Define the homogeneous Rogers-Szegö polynomial in $m$ variables for $m \geqslant 2$, denoted $\tilde{H}_{n}\left(t_{1}, t_{2}, \ldots, t_{m}\right)$, by

$$
\tilde{H}_{n}\left(t_{1}, t_{2}, \ldots, t_{m}\right)=\sum_{k_{1}+\cdots+k_{m}=n}\left(\begin{array}{c}
n \\
k_{1}, \ldots, k_{m}
\end{array}\right) t_{q}^{k_{1}} \cdots t_{m}^{k_{m}},
$$

and define the Rogers-Szegö polynomial in $m-1$ variables, denoted $H_{n}\left(t_{1}, \ldots, t_{m-1}\right)$, by

$$
H_{n}\left(t_{1}, \ldots t_{m-1}\right)=\tilde{H}\left(t_{1}, \ldots, t_{m-1}, 1\right) .
$$

The homogeneous multivariate Rogers-Szegö polynomials were first defined by Rogers [16] in terms of their generating function, and several of their properties are given by Fine [5, Section 21]. The definition of the multivariate Rogers-Szegö polynomial $H_{n}$ is given by Andrews in [1, Chap. 3, Ex. 17], along with a generating function, although there is little other study of these polynomials elsewhere in the literature (however, there is a non-symmetric version of a bivariate Rogers-Szegö polynomial [4]).

The multivariate Rogers-Szegö polynomials satisfy a recursion which generalizes (1.1), although it seems not to be very well-known, as the only proof and reference to it that the author has found is in the physics literature, in papers of Hikami [7, 8]. For any finite set of variables $X$, let $\mathbf{e}_{i}(X)$ denote the $i$ th elementary symmetric polynomial in the variables $X$. Then the Rogers-Szegö polynomials in $m-1$ variables satisfy the following recursion:

$$
H_{n+1}\left(t_{1}, \ldots, t_{m-1}\right)=\sum_{i=0}^{m-1} \mathbf{e}_{i+1}\left(t_{1}, \ldots, t_{m-1}, 1\right)(-1)^{i} \frac{(q)_{n}}{(q)_{n-i}} H_{n-i}\left(t_{1}, \ldots, t_{m-1}\right) .
$$


The sum of all $q$-multinomial coefficients of length $m$, or the generalized Galois number $G_{n}^{(m)}$, is then

$$
H_{n}(1,1, \ldots, 1)=G_{n}^{(m)}=\sum_{k_{1}+\cdots+k_{m}=n}\left(\begin{array}{c}
n \\
k_{1}, \ldots, k_{m}
\end{array}\right)_{q} .
$$

From the discussion at the beginning of Section 2, when $q$ is the power of a prime, $G_{n}^{(m)}$ is exactly the total number of flags of subspaces of length $m-1$ in an $n$-dimensional $\mathbb{F}_{q}$-vector space.

Since the number of terms in the elementary symmetric polynomial $\mathbf{e}_{i+1}\left(t_{1}, \ldots, t_{m-1}, 1\right)$ is $\left(\begin{array}{c}m \\ i+1\end{array}\right)$, then the following, our last result, follows directly from the formal identity (3.1) proved by Hikami. However, we give a proof which follows directly from Theorem 2.1, and is thus a bijective proof through the enumeration of flags in a finite vector space.

Theorem 3.1. The generalized Galois numbers satisfy the recursion, for $n \geqslant m-1$,

$$
G_{n+1}^{(m)}=\sum_{i=0}^{m-1}\left(\begin{array}{c}
m \\
i+1
\end{array}\right)(-1)^{i} \frac{(q)_{n}}{(q)_{n-i}} G_{n-i}^{(m)} .
$$

Proof. For convenience, whenever any $k_{i}<0$, we define the $q$-multinomial coefficient $\left(\begin{array}{c}n \\ k_{1}, k_{2}, \ldots, k_{m}\end{array}\right)_{q}=0$. Granting this, we have Theorem 2.1 holds for all $k_{i} \geqslant 0$. We now begin with the definition of $G_{n+1}^{(m)}$ as the sum of all $q$-multinomial coefficients, and we directly apply Theorem 2.1 to rewrite the sum, as follows:

$$
\begin{aligned}
& G_{n+1}^{(m)}=\sum_{k_{1}+\cdots k_{m}=n+1}\left(\begin{array}{c}
n+1 \\
k_{1}, \ldots, k_{m}
\end{array}\right)_{q} \\
& =\sum_{k_{1}+\cdots k_{m}=n+1} \sum_{\substack{J \subseteq\{1, \ldots, m\} \\
|J|>0}}(-1)^{|J|-1} \frac{(q)_{n}}{(q)_{n-|J|+1}}\left(\begin{array}{c}
n+1-|J| \\
k-\underline{e}_{J}
\end{array}\right)_{q} \\
& =\sum_{\substack{J \subseteq\{1, \ldots m\} \\
|J|>0}} \sum_{\substack{k=\left(k_{1}, \ldots, k_{m}\right) \\
k_{1}+\ldots+k_{m}=n+1}}(-1)^{|J|-1} \frac{(q)_{n}}{(q)_{n-|J|+1}}\left(\begin{array}{c}
n+1-|J| \\
\underline{k}-\underline{e}_{J}
\end{array}\right)_{q} \\
& =\sum_{i=0}^{m-1} \sum_{\substack{J\{1, \ldots, m\} \\
|J|=i+1}} \sum_{\substack{k=\left(k_{1}, \ldots, k_{m}\right) \\
k_{1}+\cdots+k_{m}=n+1}}(-1)^{i} \frac{(q)_{n}}{(q)_{n-i}}\left(\begin{array}{c}
n-i \\
\underline{k}-\underline{e}_{J}
\end{array}\right)_{q} \\
& =\sum_{i=0}^{m-1}\left(\begin{array}{c}
m \\
i+1
\end{array}\right)_{\substack{k_{1}^{\prime}+\cdots+k_{m}^{\prime}=n-i \\
k^{\prime}=\left(k_{1}^{\prime}, \ldots, k_{m}^{\prime}\right)}}(-1)^{i} \frac{(q)_{n}}{(q)_{n-i}}\left(\begin{array}{c}
n-i \\
\underline{k}^{\prime}
\end{array}\right)_{q} \\
& =\sum_{i=0}^{m-1}\left(\begin{array}{c}
m \\
i+1
\end{array}\right)(-1)^{i} \frac{(q)_{n}}{(q)_{n-i}} G_{n-i}^{(m)} \text {, }
\end{aligned}
$$

where the next-to-last equality follows from the fact that each index $\underline{k}^{\prime}$ may be obtained from an index $\underline{k}$ from any of the $\left(\begin{array}{c}m \\ i+1\end{array}\right)$ subsets $J$ of size $i+1$. 
By a very similar argument, we may see that in fact the recursion for the multinomial Rogers-Szegö polynomials in (3.1) also follows from Theorem 2.1.

\section{Acknowledgements}

The author thanks George Andrews and Kent Morrison for very helpful comments, and the anonymous referee for very useful suggestions to improve this paper.

\section{References}

[1] G. Andrews, The Theory of Partitions. Encyclopedia of Mathematics and its Applications, Addison-Wesley, Reading, Mass.-London-Amsterdam, 1976.

[2] R. Askey and J. Wilson. Some basic hypergeometric orthogonal polynomials that generalize Jacobi polynomials. Mem. Amer. Math. Soc., 54(319), 1985.

[3] T. Bliem and S. Kousidis. The number of flags in finite vector spaces: asymptotic normality and Mahonian statistics. J. Algebraic Combin., to appear, doi:10.1007/s10801-012-0373-1.

[4] W. Y. C. Chen, H. L. Saad, and L. H. Sun. The bivariate Rogers-Szegö polynomials. J. Phys. A, 40(23):6071-6084, 2007.

[5] N. J. Fine. Basic Hypergeometric Series and Applications. Mathematical Surveys and Monographs 27, American Mathematical Society, Providence, RI, 1988.

[6] J. Goldman and G.-C. Rota. The number of subspaces of a vector space. In Recent Progress in Combinatorics (Proc. Third Waterloo Conf. on Combinatorics, 1968), pages 75-83. Academic Press, New York, 1969.

[7] K. Hikami. Representations of motifs: new aspect of the Rogers-Szegö polynomials. J. Phys. Soc. Japan, 64(4):1047-1050, 1995.

[8] K. Hikami. Representation of the Yangian invariant motif and the Macdonald polynomial. J. Phys. A, 30(7):2447-2456, 1997.

[9] S. Hitzemann and W. Hochstättler. On the combinatorics of Galois numbers. Discrete Math., 310(24):3551-3557, 2010.

[10] V. Kac and P. Cheung. Quantum Calculus. Universitext, Springer-Verlag, New York, 2002.

[11] H. Karabulut. Distributed Gaussian polynomials as $q$-oscillator eigenfunctions. J. Math. Phys., 47(1):013508, 2006.

[12] S. Kousidis. Asymptotics of generalized Galois numbers via affine Kac-Moody algebras. Proc. Amer. Math. Soc., to appear, arXiv:1109.2546.

[13] A. J. Macfarlane. On q-analogues of the quantum harmonic oscillator and the quantum group $\mathrm{SU}(2)_{q}$. J. Phys. A, 22(21):4581-4588, 1989.

[14] K. Morrison. Integer sequences and matrices over finite fields. J. Integer Seq., $9(2): 06.2 .1,2006$. 
[15] A. Nijenhuis, A. E. Solow, and H. S. Wilf. Bijective methods in the theory of finite vector spaces. J. Combin. Theory Ser. A, 37(1):80-84, 1984.

[16] L. J. Rogers. On a three-fold symmetry in the elements of Heine's series. Proc. London Math. Soc., 24:171-179, 1893.

[17] L. J. Rogers. On the expansion of some infinite products. Proc. London Math. Soc., 24:337-352, 1893.

[18] R. P. Stanley. Enumerative Combinatorics, Vol. 1. Cambridge Studies in Advanced Mathematics, 49. Cambridge University Press, Cambridge, 1997.

[19] G. Szegö. Ein Beitrag zur Theorie der Thetafunktionen. S. B. Preuss. Akad. Wiss. Phys.-Math. Kl., 242-252, 1926.

[20] S. O. Warnaar. Rogers-Szegö polynomials and Hall-Littlewood symmetric functions. J. Algebra, 303(2):810-830, 2006. 